\title{
Adaptation of Leginon Software for Semi-Automated Recording of Electron Microscopic Images of Two-Dimensional Crystals
}

\author{
A. Cheng, J. Pulokas, C. Suloway, Y. Zhu, M. Yeager, C.S. Potter, B. Carragher \\ Center for Integrative Molecular Biosciences and Department of Cell Biology, The Scripps Research \\ Institute, La Jolla, CA 92037
}

Data collection of electron cryo-micrographs from biomolecules has been a labor-intensive process requiring manual intervention by an experienced user for long periods of time. Automated data collection systems such as Leginon [1] have demonstrated the potential for automated image acquisition from single particles [2] and helical tubes [3]. We are now adapting Leginon for semiautomated recording of images of two-dimensional (2D) crystals.

A particular advantage of 2D crystallography over single particle analysis is that the averaging provided by the crystalline lattice allows the determination of high resolution maps of particles too small $(\sim 0.5 \mathrm{~nm})$ for single particle analysis. The disadvantage is that specimens have to be tilted in order to obtain three-dimensional structural information. At high tilt angles, imperfections in specimen flatness, charging, thermal drift and vibration of the cryo-stage contribute to a loss of image resolution. These problems as well as the missing cone due to a maximum tilt angle of $\sim 60^{\circ}$ determine the final resolution that can be achieved. Furthermore, $2 \mathrm{D}$ crystals are difficult to identify because they do not have a fixed shape or size. However, their uniform optical density and subtle differences in contrast does distinguish them from the debris (Figure 1).

We have used membrane preparations enriched in gap junction plaques as a test specimen to adapt Leginon for analysis of 2D crystals [4]. This sample is particularly challenging due to the rarity of crystals and the high fraction of contaminating non-junctional material. Out of $>100$ objects in a typical grid square, only 1-3 will be crystals, and even a trained microscopist can misidentify them. Only about $1 / 10^{\text {th }}$ of the crystals will have the required resolution $(0.9-0.7 \mathrm{~nm})$ so that they can be added to the data set to contribute to the final 3D reconstruction. It is therefore not unusual for a microscopist to spend 12 to $16 \mathrm{hrs}$ searching a single grid while patiently waiting for stabilization of the cryo-specimen holder between image acquisitions. Consequently, data collection from these 2D crystals would benefit enormously from automation.

The Leginon system has been adapted as follows. First, the overall quality of the grid is assessed by automatically recording CCD images of the entire grid at a very low magnification (VLM) (60x) (Figure 1a). The VLM images are organized as a tiled mosaic, and this grid atlas is then used to navigate the grid and select grid squares suitable for data collection. The automated system then acquires an image of each selected grid square (Figure 1b) at low magnification (660x). The image of the grid square is analyzed to identify target objects that correspond to $2 \mathrm{D}$ crystals with suitable ice. A CCD image of each target is acquired at an intermediate magnification (1,700x) (Figure 1c) to further assess the presence and quality of the crystals within the area. The selection of crystals to be photographed can be performed using a mouse and a computer monitor rather than the tedious process of manually scanning the grid and viewing the specimen in search mode on the phosphor screen of the electron microscope. The acquisition of the images can then be performed without user intervention as follows. In an area just adjacent to the crystal to be photographed, focusing and corrections for astigmatism are automatically performed, and measurements are taken to ensure that the specimen is not drifting. Finally, a high magnification image $(50,000 \mathrm{x})$ of the target is recorded on film. This is necessary because the CCD camera does not have a sufficient field of view or resolution to record atomic resolution images of large $2 \mathrm{D}$ crystals. The computed diffraction pattern of the crystal (Figure 1d) shows the characteristic hexagonal unit cell of gap junction crystals.

Preliminary experiments have demonstrated that the images collected using Leginon on untilted specimens have comparable resolution to those collected using traditional manual methods. 
However, further modifications will be required to improve the image quality obtained from tilted specimens. Nevertheless, these test experiments have demonstrated the potential for using Leginon software to accelerate the recording of high resolution images of $2 \mathrm{D}$ crystals.

Some of the work presented here was conducted at the National Resource for Automated Molecular Microscopy. This work was supported by NIH NCRR17573 (C.S.P. and B.C.) and NIH NHLBI RO1 HL48908 (M.Y.). M.Y. is the recipient of a Clinical Scientist Award in Translational Research from the Burroughs Wellcome Fund.

\section{References}

[1] B. Carragher et al., J. Struct. Biol. 132 (2000) 33.

[2] I. Rouiler et al., J. Struct. Biol. 133 (2001) 102.

[3] Y. Zhu et al., J. Struct. Biol. 135 (2001) 302.

[4] V. M. Unger et al., J. Struct. Biol. 128 (1999) 98.
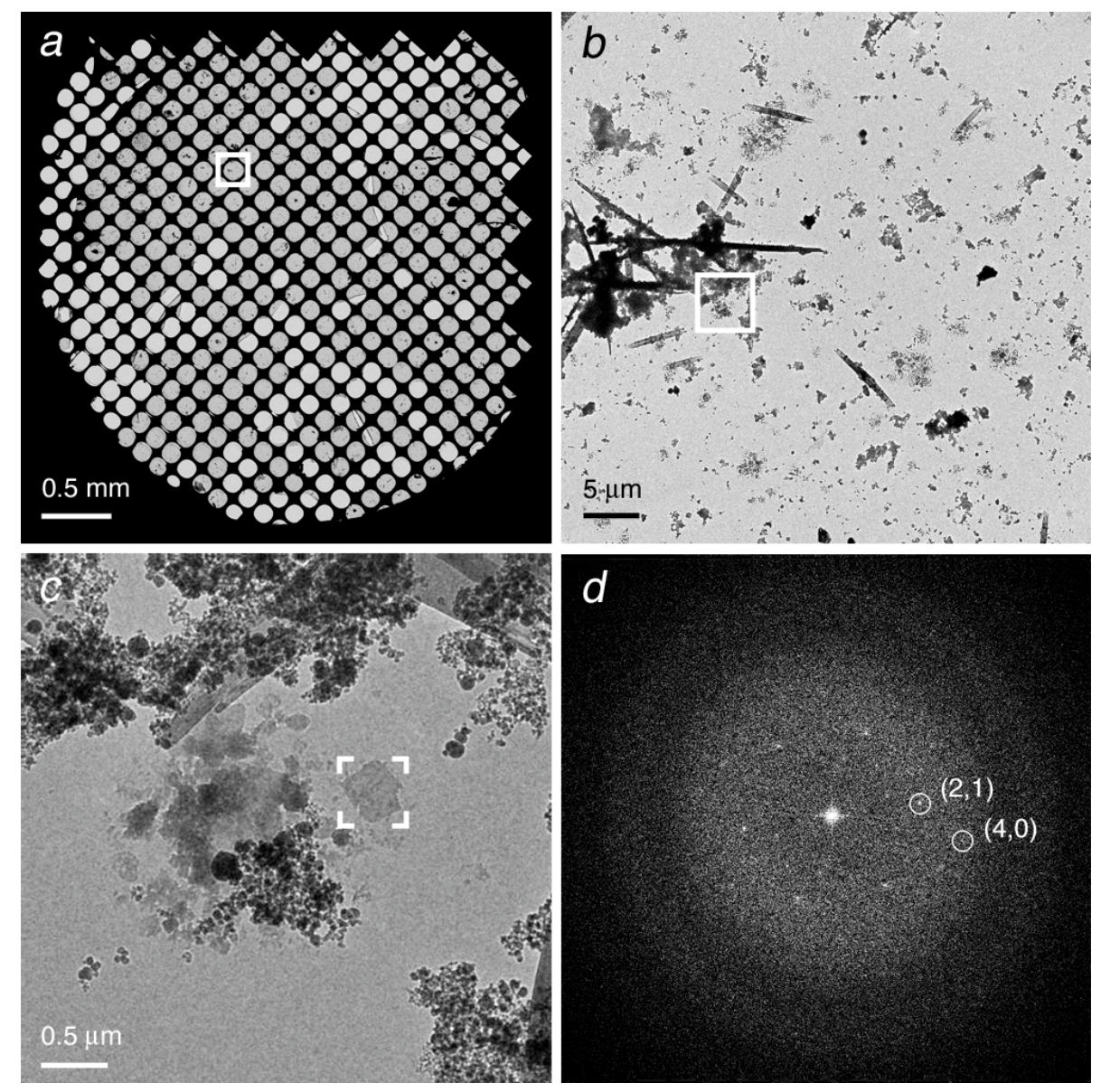

Figure 1. (a) Very low magnification image (60x) of an EM grid containing 2D gap junction crystals (b) Enlarged view (660x) of the grid square in the white box in (a). The vast majority of the material on the grid represents non-junctional membrane aggregates and crystalline precipitates. (c) Enlarged view of the region in the white box in (b). The white brackets identify a gap junction crystal. (d) Computed diffraction pattern of the 2D crystal in (c) has the characteristic unit cell of a gap junction crystal. 